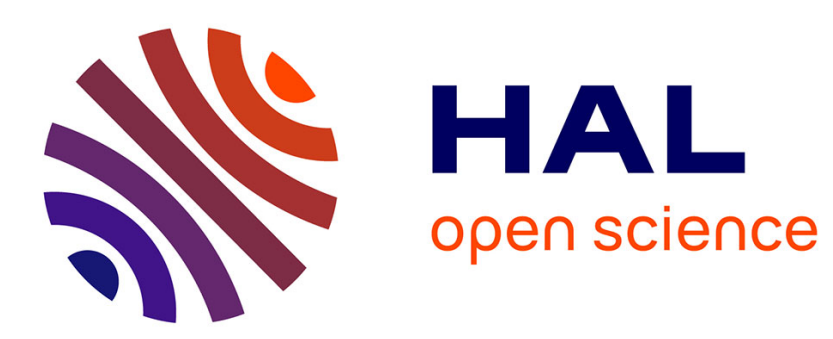

\title{
Relationships between sterol/phospholipid composition and xenobiotic transport in nematodes
}

Mickaël Riou, Isabelle Grasseau, Elisabeth Blesbois, Dominique Kerboeuf

\section{To cite this version:}

Mickaël Riou, Isabelle Grasseau, Elisabeth Blesbois, Dominique Kerboeuf. Relationships between sterol/phospholipid composition and xenobiotic transport in nematodes. Parasotol. Res, 2007, 100, pp.1125-1134. hal-00136827

\section{HAL Id: hal-00136827 https://hal.science/hal-00136827}

Submitted on 15 Mar 2007

HAL is a multi-disciplinary open access archive for the deposit and dissemination of scientific research documents, whether they are published or not. The documents may come from teaching and research institutions in France or abroad, or from public or private research centers.
L'archive ouverte pluridisciplinaire HAL, est destinée au dépôt et à la diffusion de documents scientifiques de niveau recherche, publiés ou non, émanant des établissements d'enseignement et de recherche français ou étrangers, des laboratoires publics ou privés. 


\title{
Relationships between sterol/phospholipid composition and xenobiotic transport in nematodes
}

\author{
Mickaël Riou • Isabelle Grasseau • Elisabeth Blesbois • \\ Dominique Kerboeuf
}

Received: 6 December 2006 / Accepted: 22 December 2006 / Published online: 9 February 2007

(C) Springer-Verlag 2007

\begin{abstract}
Therapeutic failure limits prophylaxis of nematode diseases and has been mainly attributed to mutations in cellular targets of anthelmintics. Besides these specific mechanisms, alterations of drug transport also occur in parasites resistant to anthelmintics and depend on both the presence of membrane pumps such as $P$-glycoprotein (Pgp) and on the lipid composition of membranes. We recently showed in the nematode Haemonchus contortus, using eggs as a model, that the total cholesterol (TC) concentration alters the transport of lipophilic molecules due to membrane pumps such as $P$-glycoprotein and the resistance to anthelmintics. The effect of TC may depend on the presence of other lipids interacting with TC. Therefore, we analysed the lipid composition and its relationship with Pgp and resistance to anthelmintics. Better correlations were found between Pgp and free cholesterol (FC) than with TC. We also showed that the relationships between lipid composition and resistance to anthelmintics or Pgp depended on the equilibrium between FC and phospholipids (PLs), mainly PLs known to be present primarily in either the external leaflets of cell membranes or the internal leaflets. The PLs phosphatidylcholine and phosphatidyleth-
\end{abstract}

M. Riou $\cdot$ D. Kerboeuf $(\bowtie)$

Multiresistances and Antiparasitic Drugs, INRA: UR1282,

Animal Infections and Public Heath, IASP,

37380 Nouzilly, France

e-mail: kerboeuf@tours.inra.fr

I. Grasseau • E. Blesbois

Quality of Gamets and Embryos, INRA: UR083 Poultry

Research,

37380 Nouzilly, France

Present address:

M. Riou

IRBI, UMR CNRS 6035, Faculty of Sciences and Techniques,

37200 Tours, France anolamine played the most significant role, but phosphatidic acid also influenced drug resistance.

$\begin{array}{ll}\text { Abbreviations } \\ \text { ABC } & \text { ATP binding cassette } \\ \text { au } & \text { arbitrary units } \\ \text { C elegans } & \text { Caenorhabditis elegans } \\ \text { DPG } & \text { diphosphoglycerids } \\ \text { FC } & \text { free cholesterol } \\ \text { H contortus } & \text { Haemonchus contortus } \\ \text { LC50 } & \text { 50\% lethal concentration } \\ \text { LPC } & \text { lysophosphatidylcholine } \\ \text { Mabs } & \text { monoclonal antibodies } \\ \text { MDR } & \text { multidrug resistance } \\ \text { R123 } & \text { rhodamine 123 } \\ \text { PA } & \text { phosphatidic acid } \\ \text { PC } & \text { phosphatidylcholine } \\ \text { PE } & \text { phosphatidylethanolamine } \\ \text { Pgp } & \text { P-glycoprotein } \\ \text { PI } & \text { phosphatidylinositol } \\ \text { PL } & \text { phospholipids } \\ \text { PS } & \text { phosphatidylserine } \\ \text { SM } & \text { sphingomyeline } \\ \text { TBZ } & \text { thiabendazole } \\ \text { TC } & \text { total cholesterol } \\ & \end{array}$

\section{Introduction}

Drug resistance can greatly limit most therapies against pathogenic agents. Besides target mutations, less specific mechanisms such as passive and active drug transport have been shown to also play a significant role in therapeutic failures. Both transport mechanisms involve lipids either 
directly as in passive drug transport through cell membranes or indirectly as in active drug transport due to efflux pumps. The role of these lipids in therapy failures has been variously investigated depending on the biological model, with most results being obtained in multi-drug-resistant tumour cells. Sterols have been identified as principal lipid agents that regulate both the transport of lipophilic molecules, such as many therapeutic agents, and the pump activities of transmembrane proteins, such as $P$-glycoproteins (Pgp; Rothnie et al. 2001; Shechter and Rossignol 1997; Zeng et al. 1999). In nematodes, resistance has been mainly attributed to specific target mutations such as changes in the polymerization of $\beta$-tubulin (Beech et al. 1994; Coles 1990; Kwa et al. 1993). We recently showed that, in addition to these mutations, Pgp activity also affects the resistance to anthelmintics of the nematode Haemonchus contortus $(\mathrm{Hc})$, a highly pathogenic trichostrongyle of sheep and goats for which numerous cases of resistance to anthelmintics have been identified around the world (Kerboeuf et al. 2002, 2003a; Riou et al. 2003). Moreover, we found that the lipid membrane environment greatly influences Pgp activity. In particular, cholesterol was shown to play a determinant role in modulating the transport of different Pgp substrates (Riou et al. 2003). Anthelmintic transport differed depending on the concentration of cholesterol in isolates having various levels of resistance. However, phospholipids (PLs) may also affect the solubilisation of xenobiotic molecules and the activities of intra-membrane proteins (Kremer et al. 2001; Pallares-Trujillo et al. 2000).

A better understanding of the lipid composition of these parasites is, thus, crucial. In nematodes, the lipids have only been analysed in the adult stages, whereas different other stages play a significant role in the biology of the parasite (Gibbons 1979). Four classes of lipids have so far been identified: PLs, sterols (mainly cholesterol), triglycerides, and free fatty acids (Kapur and Sood 1985). Until now, no study has investigated the effect of these lipids on the specific biological and biophysical features of different nematode isolates.

The aim of this study was to identify the lipid composition of different anthelmintic-susceptible or -resistant isolates Hc eggs and evaluate the relationships between these lipids (primarily sterols and PLs) and the resistance to anthelmintics of the isolates. Eggs were chosen as a model, as different methodologies can been applied to this stage, in particular, flow cytometry.

\section{Materials and methods}

Animals and parasites

Lambs were infected with $6,000 \mathrm{Hc}$ infective larvae $\left(\mathrm{L}_{3}\right)$ from each isolate. Parasites were collected from 3-month- old male "Ile de France-Charolais" lambs fed with hay and cereal. We studied four Hc isolates: two susceptible ( $\mathrm{HcS}$; HcS-WB for "Weybridge", UK, and HcS-C for "Canada") and two resistant (HcR; HcR-G for "Guadeloupe", resistant to benzimidazoles and ivermectin, and tolerant to moxidectin; and HcR-WR for "White River", South Africa, resistant to benzimidazoles and ivermectin). The eggs were isolated from faeces (Beaumont-Schwartz et al. 1987) and stored in deionised water at $4{ }^{\circ} \mathrm{C}$ for one night before use. The donor sheep were slaughtered 5 weeks after infection, following current French laws on animal experimentation. All experiments were performed with the guidelines approved by the Animal Ethics Committee of our institute.

\section{Extraction of total lipids}

Lipids were extracted from 200,000 eggs that had been broken by being frozen at $-20^{\circ} \mathrm{C}$ and then thawed The thawed samples were crushed with a Dual tissue microgrinder (with teflon pestle, Fisher-Scientific, Elancourt, France), and the total lipids were extracted with a chloroform/methanol solution (v/v, 50:50, VWR International, Pessac, France), as described by Riou et al. (2003). The resulting dried extract was then dissolved in $50 \mu \mathrm{l}$ of isopropanol for biochemical assays or $50 \mu \mathrm{l}$ of chloroform for lipid class analyses.

\section{Determination of total cholesterol and PL concentrations}

The total cholesterol concentration was determined using the cholesterol oxidase method (RTU kit, BioMérieux, Marcy l'Etoile, France). After the enzymatic transformation of cholesterol in quinoneimine, the pink colour was quantified by measuring the absorbance at $500 \mathrm{~nm}$. The cholesterol concentration (ng/1,000 eggs) was determined from a calibration curve derived from the kit reference cholesterol solution (Riou et al. 2003).

The PL concentration was determined using the PL hydrolase method (PAP150 kit, BioMérieux). After the enzymatic transformation of the PLs in quinoneimine, the pink colour was quantified by measuring the absorbance at $505 \mathrm{~nm}$. The PL concentration (ng/1,000 eggs) was determined from a calibration curve derived from the kit reference PLs.

Thin-layer chromatography analysis of lipids

\section{Total lipids}

The following presents the total egg lipids analysed as described by Douard et al. (2000). Briefly, the lipid classes were separated by thin-layer chromatography on 
silica rods. The rods were run in hexane/ethyl-ether/acetic acid $(\mathrm{v} / \mathrm{v} / \mathrm{v}, 87: 13: 0.75)$ for $20 \mathrm{~min}$. The detection and quantification were made using the iatroscan system (Iatron, Tokyo, Japan). The chromatograms were compared to the following standards: sterol esters (cholesterol oleate, 10\%, Sigma-Aldrich), triglycerides (tripalmitine, $10 \%$, Sigma-Aldrich), sterols (cholesterol, 20\%, SigmaAldrich), and PLs [phosphatidylcholine (PC), 60\%, Sigma-Aldrich].

\section{Phospholipids}

Egg PLs were analysed as described by Surai et al. (2000). Briefly, PL classes were separated by thin-layer chromatography on silica rods. They were first run in acetone for $20 \mathrm{~min}$ and then in chloroform/acetic acid/water $(\mathrm{v} / \mathrm{v} / \mathrm{v}$, $80: 35: 3)$ for $45 \mathrm{~min}$. The detection and quantification were made using the iatroscan system (Iatron). The chromatograms were compared to the following standards (SigmaAldrich): phosphatidic acid (PA, 10\%), diphosphoglycerid (DPG, 10\%), phosphatidylinositol (PI, 5\%), PC (30\%), phosphatidylserine (PS, 5\%), sphingomyeline (SM, 10\%), lysophosphatidylcholine (LPC, 10\%), and phosphatidylethanolamine (PE, 20\%).

Pgp activity and localisation

\section{Pgp activity}

The Pgp activity was expressed as the number of Pgp $(+)$ eggs revealed by UIC2 mAbs (Immunotech, Marseille, France) staining estimated by flow cytometry analyses after the R123-stimulation that had given a maximum expression of Pgp activity. These results were taken from Kerboeuf et al. (2003b). The UIC2 mAbs recognise an epitope associated with a specific active Pgp conformation induced by drugs, thus, revealing the pump activity (Mechetner and Roninson 1992).

\section{Localisation of Pgp by transmission electron microscopy}

The egg samples were fixed in a mixture of $0.2 \%$ glutaraldehyde and $4 \%$ paraformaldehyde in phosphatebuffered saline ( $\mathrm{pH} 7.4$ ) by incubation overnight at $4^{\circ} \mathrm{C}$ and were then embedded in 100\% epoxy resin as described by Riou et al. (2005a). Each grid was incubated in a drop of 1:2 diluted Pgp-specific mAb UIC2. Antibody binding was detected by incubation with 1:10 diluted goat anti-mouse IgG secondary antibodies conjugated to $10-\mathrm{nm}$ gold particles. The grids were then observed with a CM10 electron microscope (Philips, Eindhoven, Pays-Basthe, The Netherlands). The images were acquired by an analysis software (Soft Imaging System, Germany).

\section{Measurement of anthelmintic resistance}

The level of resistance of the four isolates were taken from Riou et al. (2003) using in vitro egg hatch assays according to Beaumont-Schwartz et al. (1987; Riou et al. 2003). Briefly, eggs were incubated for $48 \mathrm{~h}$ at $22^{\circ} \mathrm{C}$ with thiabendazole (TBZ) concentrations from 0.02 to $0.08 \mu \mathrm{g} /$ $\mathrm{ml}$ for the susceptible isolates and from 0.24 to $1.26 \mu \mathrm{g} / \mathrm{ml}$ for the resistant isolates. TBZ was chosen as a model compound for benzimidazole anthelmintics. The hatching rates were compared to those of control eggs treated with deionised water and TBZ only. LC50 values were calculated after the log-probit transformation of the data.

\section{Analysis of xenobiotic transport}

Xenobiotic transport was determined by the uptake of rhodamine 123 (R123 Sigma-Aldrich, Saint Quentin, France), a fluorescent substrate specific for Pgp pumps. Thirty thousand eggs were incubated with $200 \mu$ l of R123 $(0.5 \mu \mathrm{g} / \mathrm{ml})$ at room temperature for $15 \mathrm{~s}$, a duration determined as long enough to obtain the maximum uptake (Fig. 1). Twenty-eight milliliters of water was then added, and the green fluorescence was immediately measured by flow cytometry. The results were expressed in arbitrary units (au) calculated as the difference between the fluorescence of eggs with no R123, and the fluorescence of eggs stained with R123, thus, eliminating any native green fluorescence that differed between isolates (Kerboeuf et al. 1999, 2002).

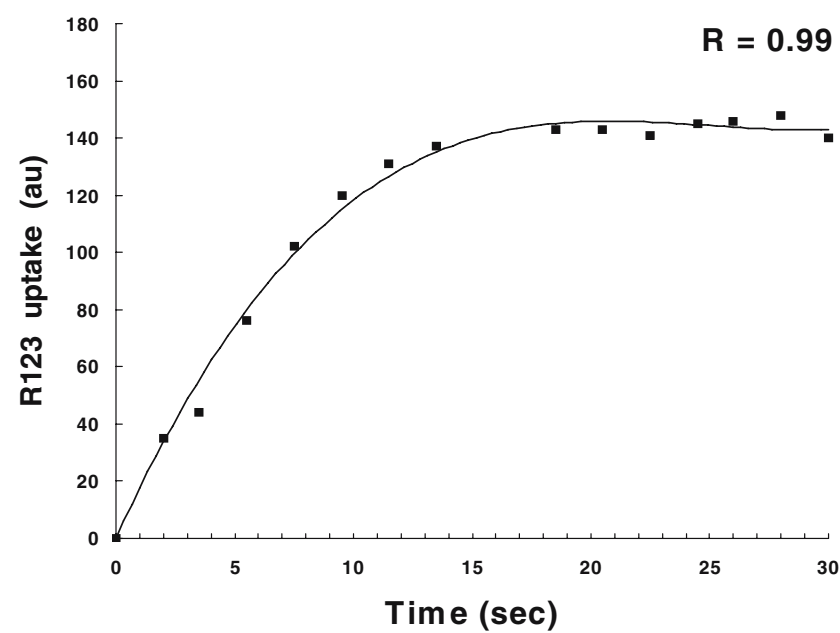

Fig. 1 Kinetics of R123 uptake (arbitrary units, au) by H. contortus. Guadeloupe egg isolate $(\mathrm{HcR}-\mathrm{G})$ chosen to determine the shortest contact time required for the maximum uptake of R123 by Pgp. The maximum uptake was obtained after $15 \mathrm{~s}$ 
Calculations and statistical analyses

Three replicates were made for each treatment and for the different parameters. The results from each identified lipid peak of the chromatograms were converted into $\mathrm{ng} / 1,000$ eggs according to the total cholesterol and PL concentrations. The membrane fluidity was estimated from the ratio free cholesterol/PLs. The results were analysed by variance analysis (ANOVA), and the means were compared using Student's $t$ tests or regression analyses (Prism 4, GraphPad software, USA).

\section{Results}

Biochemical determination of cholesterol and PL contents

The mean total cholesterol concentration determined by the cholesterol oxidase method was significantly higher (Table 1 , $P<0.05$ ) in eggs of the $\mathrm{HcR}-\mathrm{G}$ isolate than of the $\mathrm{HcS}-\mathrm{WB}$, $\mathrm{HcS}-\mathrm{Can}$, and HcR-WR, whereas for these three isolates, it was not significantly different. There was no significant difference in the total PL contents between susceptible and resistant isolates (Table 1).
The thin-layer chromatography of lipids allowed us to distinguish free cholesterol from its derivatives, mainly sterol esters that made up between 1 and $5 \%$ of the total lipids for all isolates. The mean free-cholesterol concentration in eggs was significantly higher in the two resistant isolates than in the susceptible isolates. The two susceptible isolates did not differ, but differed from the HcR-WR isolate, which was also different from the $\mathrm{HcR}-\mathrm{G}$ isolate (Table 1, $P<0.05$ ). The sterol ester concentrations differed between isolates, although there was no relationship with the level of resistance.

Two other major lipid classes were observed: the triglycerides, representing $70 \%$ of the lipids, and the PLs, representing $20 \%$ (Fig. 2a). Eight PLs were identified (Fig. 2b): PC (49\% of the total PLs), PE and PI (37\%), PA and DPG (7\%), SM and LPC (5\%), phosphatildylserine (PS, 2\%). Each isolate had its own PL profile.

\section{Immunogold staining of Pgp}

The transmission electron microscopy analysis (TEM, Fig. 3) allowed us to identify the three layers of nematode eggshells (vitellin, chitinous, and phospholipidic). The immunogold staining with UIC2 showed that Pgp staining

Table 1 Sterol and phospholipid compositions of H. contortus eggs (ng/1,000 eggs)

\begin{tabular}{|c|c|c|c|c|}
\hline \multirow[b]{2}{*}{ Lipid concentration ng/1,000 eggs } & \multicolumn{4}{|l|}{ Isolates } \\
\hline & HcS-WB & $\mathrm{HcS}-\mathrm{Can}$ & HcR-WR & $\mathrm{HcR}-\mathrm{G}$ \\
\hline \multicolumn{5}{|l|}{ Biochemical analyses } \\
\hline Total cholesterol & $53.28 \pm 13.42$ & $74.67 \pm 4.51$ & $64.67 \pm 2.08$ & $114.67^{\mathrm{a}, \mathrm{b}} \pm 10.07$ \\
\hline Total phospholipids & $804.67 \pm 0.58$ & $799.67 \pm 4.93$ & $796.67 \pm 6.66$ & $785.00 \pm 5.29$ \\
\hline \multicolumn{5}{|l|}{ Thin layer chromatograms } \\
\hline Free cholesterol & $44.50 \pm 11.21$ & $54.51 \pm 3.29$ & $64.67^{\mathrm{b}} \pm 2.08$ & $86.93^{\mathrm{b}, \mathrm{c}} \pm 7.63$ \\
\hline Sterol esters & $8.78 \pm 2.21$ & $20.15 \pm 1.22$ & $0.00 \pm 0.00$ & $27.74 \pm 2.44$ \\
\hline PA and DPG & $37.24 \pm 0.03$ & $83.79 \pm 0.52$ & $92.09 \pm 0.77$ & $89.21 \pm 0.60$ \\
\hline $\mathrm{PE}$ and PI & $231.24 \pm 0.17$ & $347.17 \pm 2.14$ & $351.39 \pm 2.94$ & $237.18 \pm 1.60$ \\
\hline PS & $40.21 \pm 0.03$ & $0.00 \pm 0.00$ & $0.00 \pm 0.00$ & $0.00 \pm 0.00$ \\
\hline $\mathrm{PC}$ & $451.78 \pm 0.32$ & $368.71 \pm 2.27$ & $329.20 \pm 2.75$ & $427.72 \pm 2.88$ \\
\hline LPC & $44.21 \pm 0.03$ & $0.00 \pm 0.00$ & $23.99 \pm 0.20$ & $17.85 \pm 0.12$ \\
\hline $\mathrm{SM}$ & $0.00 \pm 0.00$ & $0.00 \pm 0.00$ & $0.00 \pm 0.00$ & $13.04 \pm 0.12$ \\
\hline Ratio FC/PL & $0.055 \pm 0.014$ & $0.068 \pm 0.008$ & $0.081^{\mathrm{b}} \pm 0.003$ & $0.146^{\mathrm{b}} \pm 0.012$ \\
\hline
\end{tabular}

The mean free cholesterol concentration in eggs increased significantly with the resistance of isolates $(P<0.05)$. The sterol ester concentrations differed between isolates but showed no relationship with the level of resistance. Each isolate has its own PL profile. The FC/PL ratio, representative of the membrane fluidity, increased significantly with the level of resistance $(P<0.05)$. Statistical analyses: The differences in free cholesterol concentration were reported in this table, as a significant difference was observed between resistant and susceptible isolates. The differences in sterol esters and PLs were not reported in this table as they mainly came from individual specificities rather than from the level of resistance. Means \pm SD for the three measurements

HcS-WB Susceptible Haemonchus contortus Weybridge, HcS-Can susceptible Haemonchus contortus Canada, HcR-WR resistant Haemonchus contortus White River, and $H c R-G$ resistant Haemonchus contortus Guadeloupe, $P A$ phosphatidic acid, DPG diphosphoglycerids, $P E$ phosphatidylethanolamine, PI phosphatidylinositol, $P S$ phosphatidylserine, $P C$ phosphatidylcholine, $S M$ sphingomyeline, $L P C$ lysophosphatidylcholine

${ }^{\mathrm{a}}(\mathrm{HcS}-\mathrm{WB}=\mathrm{HcS}-\mathrm{Can}=\mathrm{HcR}-\mathrm{WR})<\mathrm{HcR}-\mathrm{G}$

${ }^{\mathrm{b}}$ Significant effect $(P<0.05)$

${ }^{\mathrm{c}}(\mathrm{HcS}-\mathrm{WB}=\mathrm{HcS}-\mathrm{Can})<\mathrm{HcR}-\mathrm{WR}<\mathrm{HcR}-\mathrm{G}$ 
Fig. 2 a, b examples of iatroscan lipid profiles of $H$. contortus eggs. a Profile of lipid classes: four lipid classes were observed in all isolates - sterol esters, triglycerides, cholesterol, and phospholipids. b Profile of phospholipid classes: eight phospholipids were observed in all isolates-phosphatidic acid $(P A)$, diphosphoglycerid $(D P G)$, phosphatidylethanolamine $(P E)$ and phosphatidylinositol $(P I)$, phosphatidylserine $(P S)$, phosphatidylcholine $(P C)$, sphingomyeline $(S M)$, and

lysophosphatidylcholine $(L P C)$. Values in parentheses denote retention time
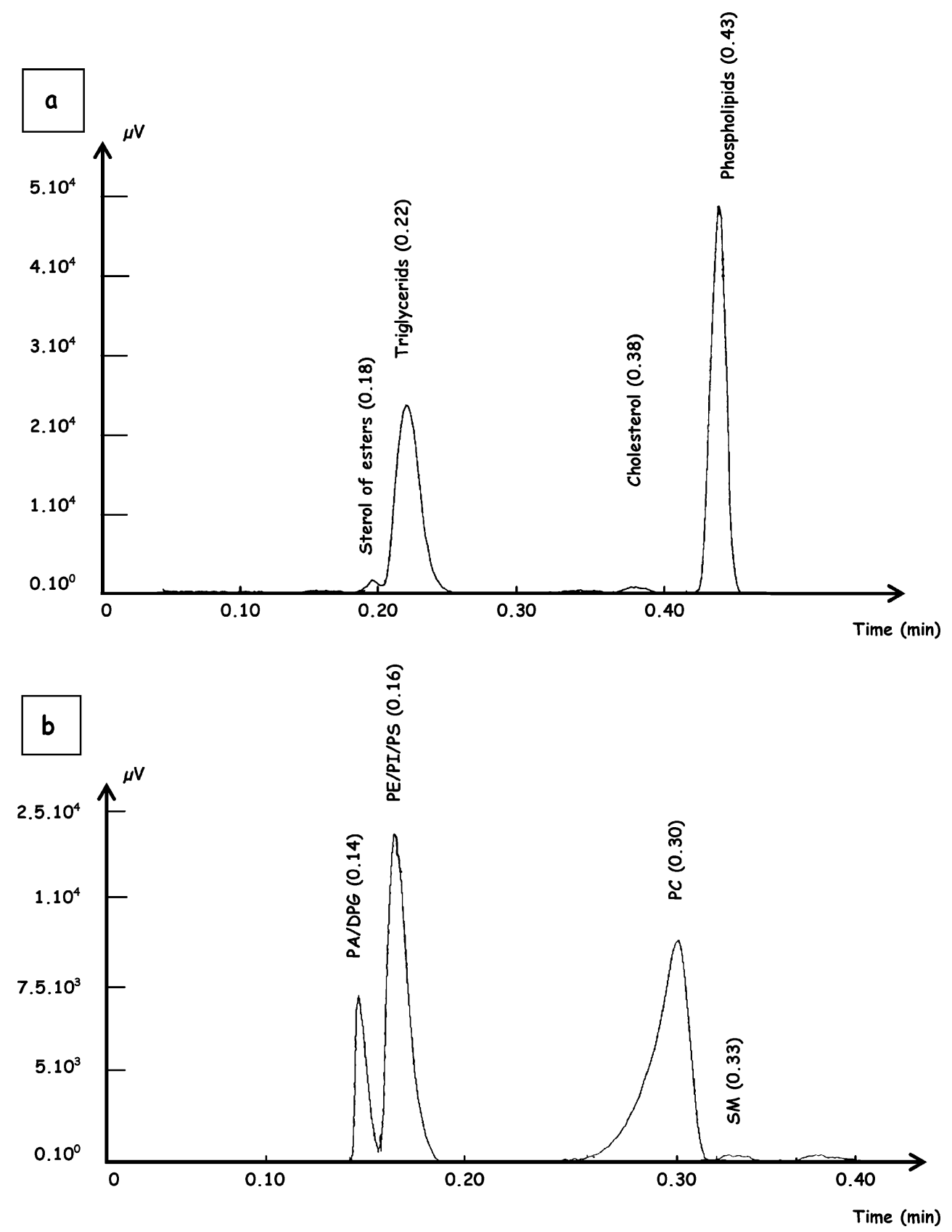

was present in all three layers of eggshells The isotypic control showed that there was no unspecific staining.

Effect of sterols on xenobiotic transport

\section{Effect on resistance to anthelmintics}

We observed a highly significant Boltzmann sigmoidal regression ( $r=0.99, P<0.01)$ between the level of resistance estimated by the LC50 for TBZ and the free cholesterol content (Fig. 4a). The Boltzmann model allowed us to define the limit of free cholesterol required for a change in resistance (63 $\mathrm{ng} / 1,000 \mathrm{eggs})$.

\section{Effect on staining with anti-Pgp mAbs}

Pgp staining, estimated by the number of eggs binding UIC2 mAbs after stimulation with the Pgp-specific substrate R123, significantly correlated with the amount of free cholesterol $(r=0.98, P<0.02$, Fig. 4b). However, the relationship was non-linear, with the highest Pgp staining being observed for a free-cholesterol concentra- 
Fig. 3 The three layers of nematode eggshells are visible: vitellin, chitinous, and lipid/protein layer. UIC2 immunogold staining of Pgp in eggshells (Haemonchus contortus Guadeloupe resistant isolate). UIC2 $\mathrm{mAb}$ is specific to the active conformation of Pgp. The clustering of immunogold particles formed well-defined black, round staining. No staining was observed for the two isotypic controls. Eg Eggshell, Ext extracellular medium, Int intracellular medium (magnification, $21,000 \times$

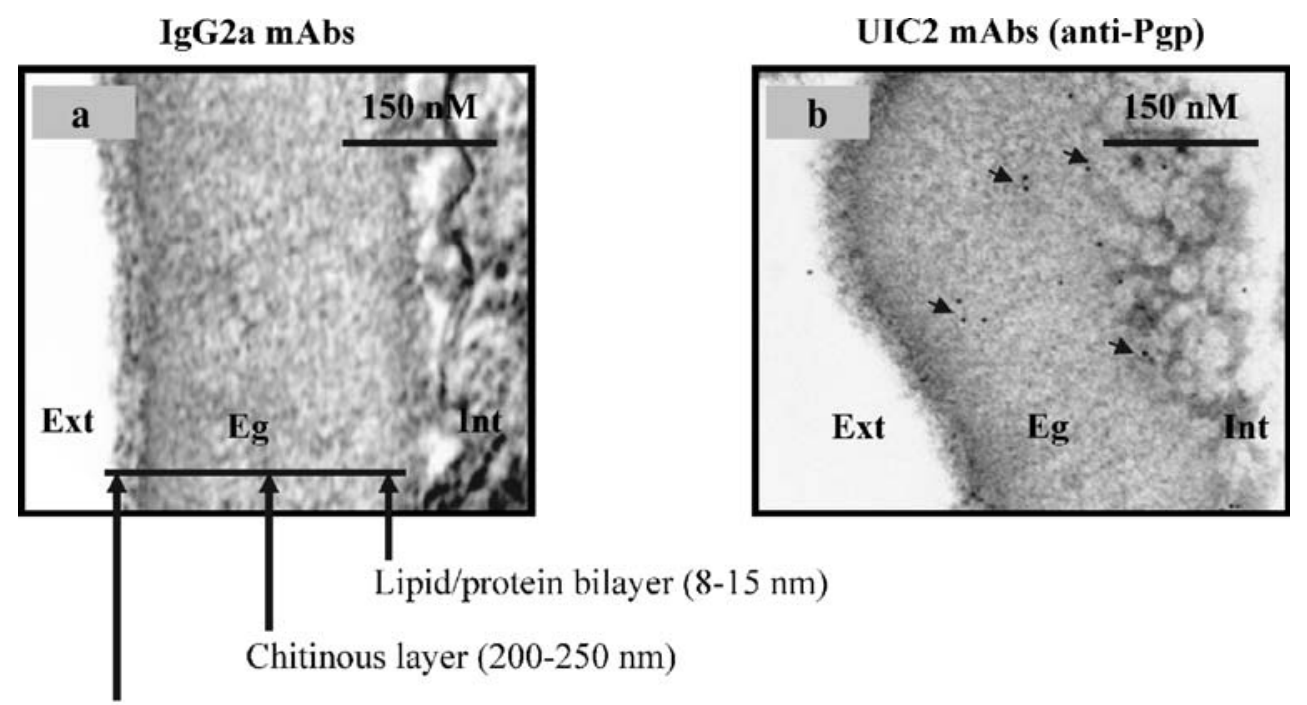

Vitellin layer $(45-70 \mathrm{~nm})$ tion of about $63 \mathrm{ng} / 1,000$ eggs. The number of eggs exhibiting Pgp was lower when the free cholesterol content was high $(\mathrm{HcR}-\mathrm{G})$.

\section{Effect on R123 uptake}

The level of green fluorescence due to the uptake of R123 seemed also to be correlated with the free cholesterol concentration. As with the Pgp staining, we observed a non-linear relationship with the highest level of green fluorescence being seen for about $63 \mathrm{ng}$ of free cholesterol/ 1,000 eggs. A low R123 uptake seemed to be associated with a high free-cholesterol concentration, although this was not significant $(r=0.92$, Fig. $4 b)$.

\section{Effect of PLs on xenobiotic transport}

No significant relationship was found between LC50 or Pgp staining and the total PL concentration. However, a significant decrease in Pgp, assessed by the number of UIC2 (+) eggs, was observed as the ratio PC/PC+PE and PI, estimating the equilibrium between the composition of the two membrane leaflets, became higher $(r=0.95, P<0.05$, Table 2). No significant correlation was found between R123 uptake and PL composition.

Relationships between the ratio "free cholesterol/PLs" and xenobiotic transport

The level of resistance significantly increased $(r=0.95$, $P<0.05$ ) with the free cholesterol to total PL ratio (Table 1). The relationship was better $(r=0.096, P<0.05)$ when considering the free cholesterol to $\mathrm{PC} / \mathrm{PE}$ and PI ratio (Table 2).
There was also a significant relationship $(r=0.99, P<0.01)$ between Pgp staining and free cholesterol to PA and DPG ratio (Table 2).

No significant relationship was found between PL composition and R123 uptake. A significant relationship $(r=0.95, P<0.05)$ between R123 uptake and Pgp activity was shown with a lower uptake in susceptible isolates than in resistant isolates (Table 2).

\section{Discussion}

In several nematode species, four major lipid classes have been identified in adult stages (Kapur and Sood 1985, 1987), but accurate determinations of the lipid composition have not been carried out. For the egg stages, Clarke et al. (1967) showed that the eggshells of another two nematodes (Ascaris spp. and Heterodera rostochiensis) contain 7\% lipids. The physiological roles of the different lipids in nematodes remain unclear. In this paper, we studied the sterol and PL composition of Hc eggs and their possible roles in xenobiotic transport.

Four main classes of lipids were identified as already described in the adult stages of other nematode species: triglycerides, sterols, sterol esters, and PLs, with a predominance of triglycerides. We did not detect lipids specific to Hc, unlike for the adult stage of Ascaris spp. (Mehlhorn 2001). The overall lipid composition of nematode eggs was found to be very similar to that of many vertebrate cells, except for the very high levels of triglycerides that are found as reserves in non-feeding nematode stages, such as eggs (Kapur and Sood 1985, 1987; Mehlhorn 2001). Triglycerides are not present in membranes and envelopes. 

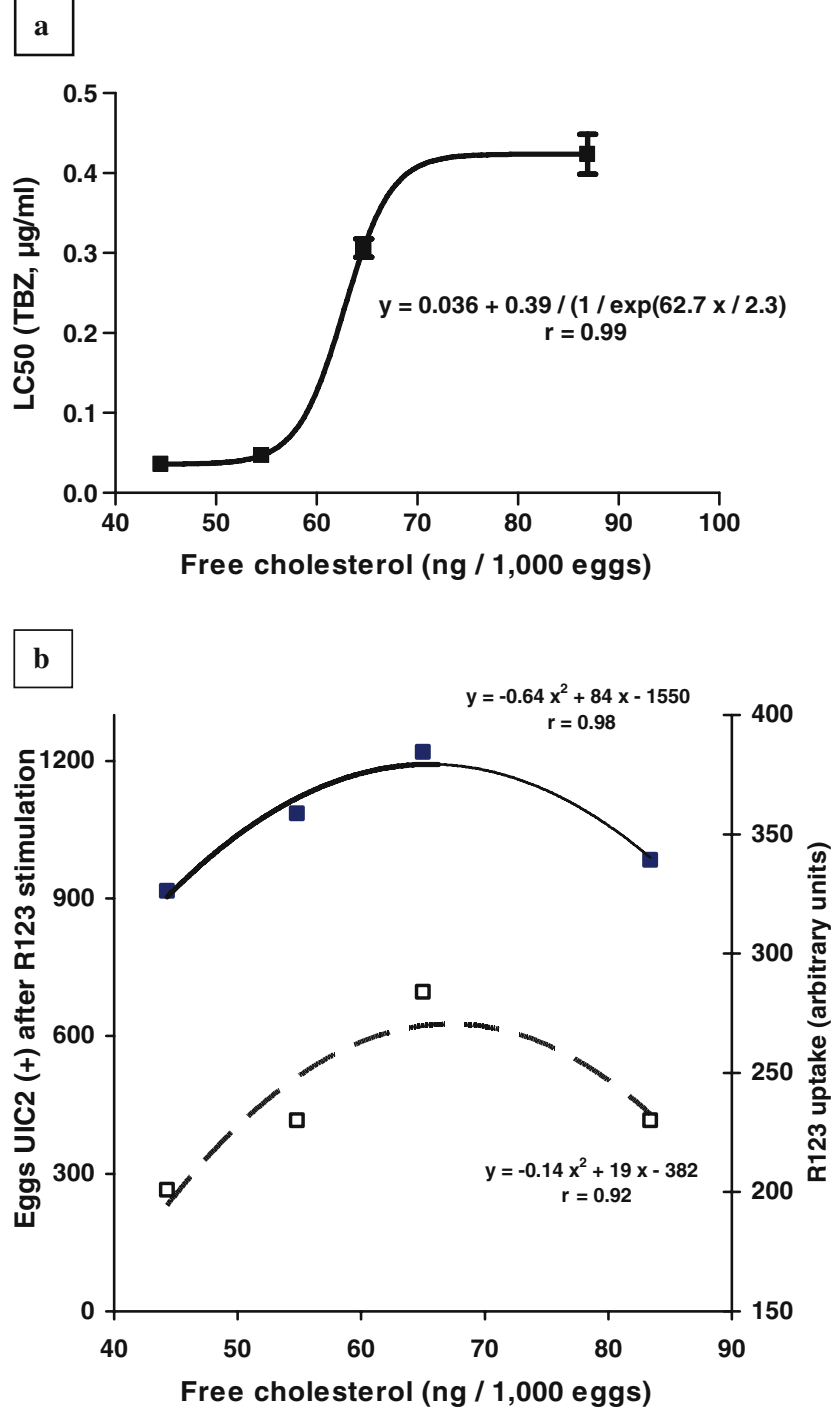

Fig. 4 a, b The free cholesterol concentration influenced anthelmintic resistance (TBZ; a) and the number of Pgp-active eggs [UIC2 (+) eggs; b) in $H$. contortus eggs from different isolates. a A highly significant Boltzmann sigmoidal regression was observed between the level of resistance estimated from the LC50 to TBZ and the free cholesterol content $(P<0.01)$. b The relationship between the number of UIC2 (+) eggs and the free cholesterol content showed an optimum of Pgp activity at a free cholesterol concentration of about $63 \mathrm{ng} / \mathrm{ml}$ $(P<0.05)$. R123 uptake by eggs showed a similar, although nonsignificant, relationship with free cholesterol concentration. Mean \pm SD for the three measurements

Sterols, particularly cholesterol, are major component of cell membranes (Kurzchalia and Ward 2003). Nematodes either synthesise little or no cholesterol, absorbing it from their hosts. Nevertheless, cholesterol is the precursor of many cholesterol derivatives that take part in their physiology (Chitwood and Lusby 1991; Hieb and Rothstein 1968; Sarwal et al. 1989). Our biochemical analyses confirm the presence of detectable amounts of cholesterol as previously observed (Riou et al. 2003). Its role remains to be better investigated, as no significant difference was observed for total cholesterol between isolates except for HcR-G that contained significantly more total cholesterol than the three other isolates.

Thin layer chromatography gave us new information on free cholesterol and sterol esters. Free cholesterol was significantly more abundant in resistant isolates than in susceptible isolates. This was also highly positively correlated with the level of resistance defined by the LC50 for TBZ. The eggs of both resistant isolates contained more free cholesterol. Cholesterol, free cholesterol, and sterol esters have different functions (Visconti et al. 1999) and locations in cells (Matyash et al. 2001; Ohvo and Slotte 1996). In vertebrates, free cholesterol is transported towards the membranes where it is either transformed into sterol esters in the membrane or stored in specific membrane micro-domains such as rafts or caveolae (Chao et al. 2005; Flesch et al. 2001; Shio et al. 1979). In nematodes, Pgp function seems to depend on the lipid environment of the eggshells. As in other models, free cholesterol may modulate resistance by decreasing the membrane electrostatic charge and limiting the drug-lipid interaction and drug influx. For example, high cholesterol concentrations have been found in multi-drug resistance (MDR) cells (Oram et al. 2003; Pallares-Trujillo et al. 2000; Raggers et al. 2000). In eggs with active Pgp, we show that the number of Pgp increased with free cholesterol concentration up to a maximum (63 ng/1,000 eggs), whereas it decreased when the free cholesterol concentration became greater than this maximum. This observation could be compared to the relative toxicity of cholesterol described in vertebrate cells. A too high free-cholesterol content in membranes is toxic, and therefore, the free cholesterol and ester sterols are stored in lipid droplets in cells (McGlynn et al. 2004).

The data previously obtained on the presence and activity of Pgp (Kerboeuf et al. 2003b) were completed in this study by the direct study of xenobiotic uptake to rely on the lipid composition with specific biological features of xenobiotic transport. Four Hc isolates with different levels of resistance to anthelmintics were studied. R123 was used as a model of lipophilic molecule. The free-cholesterol concentration does not correlate significantly with the rate of R123 uptake that provides a direct estimation of xenobiotic transport. However, the relationship between R123 uptake and free cholesterol was similar to that of the Pgp number and free cholesterol. These results suggest that R123 uptake is indirectly affected by changes in Pgp activity that itself depends on the free-cholesterol concentration. This is consistent with other studies showing a similar relationship with an optimum cholesterol concentration being observed for the highest Pgp activity in reconstituted liposomes (Klein et al. 1995; Osterberg et al. 2001). The lack of very significant correlation between free cholesterol and R123 uptake could be due to the complex 
Table 2 Relationships between lipid composition of $H$. contortus eggs, number of Pgp in the active conformation, xenobiotic transport, and resistance to anthelmintics

\begin{tabular}{|c|c|c|c|c|c|}
\hline$Y$ & $X$ & Regression & $\mathrm{fd}$ & $r$ & $P<$ \\
\hline UIC2 (+) eggs after R123 stimulation ${ }^{a}$ & $\mathrm{PC} / \mathrm{PC}+\mathrm{PE}$ and $\mathrm{PI}$ & $y=-1,397 x+1,855$ & 2 & 0.95 & 0.05 \\
\hline $\mathrm{LC} 50(\mathrm{TBZ}, \mu \mathrm{g} / \mathrm{ml})^{\mathrm{a}}$ & Free $\mathrm{C} /(\mathrm{PC} / \mathrm{PE}$ and $\mathrm{PI})$ & $y=7.0 x \times-043$ & 2 & 0.96 & 0.05 \\
\hline UIC2 (+) eggs after R123 stimulation ${ }^{\mathrm{a}}$ & Free $\mathrm{C} / \mathrm{PA}$ and $\mathrm{DPG}$ & $y=-101 x+1,303$ & 2 & 0.99 & 0.01 \\
\hline R123 uptake (arbitrary units) & UIC2 $(+)$ eggs after R123 stimulation $^{\text {a }}$ & $y=0.249-25.76$ & 2 & 0.95 & 0.05 \\
\hline
\end{tabular}

The Pgp activity estimated by the number of UIC2 $(+)$ eggs depends on the equilibrium between the different phospholipids in the membranes and is negatively influenced by the free cholesterol to PA and DPG ratio. The amount of R123 taken up is directly proportional to the Pgp activity. The resistance to anthelmintics increased with the free cholesterol to the phospholipid ratio. A more significant positive relationship was obtained with the free cholesterol to PC/PE and PI ratio.

${ }^{a}$ Taken from the reference literatures (Kerboeuf et al. 2003a,b; Riou et al. 2003)

$P A$ Phosphatidic acid, DPG diphosphoglycerids, $P E$ phosphatidylethanolamine, $P I$ phosphatidylinositol, $P C$ phosphatidylcholine, R123 rhodamine 123, TBZ thiabendazole, LC50 lethal concentration $50 \%$

structure of eggshells compared to cell membranes or liposomes. Eggshells consist of three layers: an outer vitellin layer, a middle chitinous layer, and an inner PL layer (Mansfield et al. 1992; Mehlhorn 2001). Pgp are present in these peculiar structures. Using immunogold TEM analyses with UIC2 mAbs that recognised an external epitope of the first extracellular loop present only in active Pgp, we investigated the distribution of Pgp in the eggshells of Hc and confirmed that Pgp with the active conformation was present in all three layers of eggshells, as previously reported by Riou et al. (2005a). Nevertheless, these peculiar structures could affect the relationships between xenobiotic or drug transport and lipid environment. Further investigations are needed to understand the exact role of Pgp in these structures, and how they are transported from their place of synthesis to the most external parts of the eggs.

The specific PL analysis allowed us to identify four major PLs: PC, PI, PS, and PE, as seen for other eukaryotes (Shechter and Rossignol 1997; Douard et al. 2000; Surai et al. 2000) or for adult nematodes such as Haemonchus or Onchocerca gibsoni (Kapur and Sood 1985; Maloney and Semprevivo 1991). The eggs of Hc have a PL composition very similar to that of mammals. The major PL was PC, a component of the external membrane leaflet in eukaryotes; PE and PI, respectively, components of the internal and external leaflet PC, can interact with drugs, leading to membrane deformation and drug penetration (Berleur et al. 1985). The PC to PE and PI ratio affects the stability of the membrane and decreases with drug resistance in tumour cells (Pallares-Trujillo et al. 2000). We found similar relationships in nematode eggs.

In vertebrates, the PE and PS present in the internal leaflet have to be actively transported to the external leaflet by the ATP-dependant pump proteins, amongst which are Pgp (Pohl et al. 2002). PLs also increase the affinity of ATPases for ATP and maintain them in an active confor- mation (Laird et al. 1986a,b). ATP hydrolysis increases as the $\mathrm{PC}$ to $\mathrm{PC}+\mathrm{PE}$ ratio increases. Our study has shown that eggs having the lowest $\mathrm{PC}$ to $\mathrm{PC}+\mathrm{PE}$ and $\mathrm{PI}$ ratio have the highest Pgp activity. This may correspond to the best equilibrium between the external and the internal composition of the two membrane leaflets, as previously suggested for vertebrate cells (Shechter and Rossignol 1997). This relationship also depends on the cholesterol concentration that modulates the ATPase activity of Pgp (Garrigues et al. 2002; Ghetie et al. 2004; Rothnie et al. 2001). The relationship between PLs and the cholesterol according to their respective concentrations in the eggshells remains to be better investigated.

Hc eggs also contain significant amounts of PA and DPG. PA is an anionic PL with at least three identified biochemical properties. It can bind xenobiotic molecules, such as doxorubicin (Goormaghtigh et al. 1980) or cysplatin (Speelmans et al. 1996), and can cause cytotoxicity without penetrating inside the cell by inducing disorder in the acyl chains. This occurs when the temperature is closed to that of the transition phase. PA also helps capture proteins by forming recruitment sites for cytosolic molecules and then translocating them to the plasma membrane (Manifava et al. 2001; Radhakrishna and Donaldson 1997). It has been suggested as a regulator of membrane transport. Finally, PA is also involved in the synthesis or "degradation" of other PLs. Our study has shown that the free cholesterol to PA and DPG ratio was higher in susceptible eggs, with the smallest amount of active Pgp. PA may, thus, act either by decreasing the translocation of Pgp to the external membranes or by increasing the drug toxicity.

Sterols interact with the movements of the membrane PLs and, thus, modulate the physical properties of the membrane such as fluidity (Klein et al. 2003; Shechter and Rossignol 1997; Sinicrope et al. 1992). Membrane fluidity has been shown to vary according to changes in the $\mathrm{FC}$ to 
PLs ratio. Cholesterol plays a major role in these changes but the concentration and nature of the PLs, particularly the degree of non-saturation of the fatty acids, could also alter the physical properties of the membrane. The more unsaturated the PLs, the more fluid the membranes are (Shechter and Rossignol 1997). In nematode eggs, xenobiotic efflux varied with the experimental alteration of the cholesterol content (Riou et al. 2003). Moreover, during the embryonation of eggs, the lipid composition of eggshells is naturally altered and, at the same time, resistance increased. We previously suggested that the observed changes during embryonation could be due to the modification of the membrane fluidity (Riou et al. 2005b). In the present study, we observed, in physiological conditions, a significant positive relationship between the free cholesterol to the PLs ratio $(\mathrm{FC} / \mathrm{P})$ and the LC50 that could be interpreted as an increasing resistance with a decrease in membrane fluidity. Our results also showed that the optimum cholesterol concentration (mean $=63 \mathrm{ng} / 1,000$ eggs) was associated with the highest number of Pgp with the active conformation. This suggests that the distribution of cholesterol within the membranes may be of some importance for its effects on Pgp conformation. In an MDR cell, active Pgp has been found up-regulated in membrane micro-domains and characterised by a moderately reduced fluidity and high cholesterol content (McConnell and Vrljic 2003). Certain membrane proteins are trapped in such micro-domains, and their interactions with lipids confer specific properties on them (Crane and Tamm 2004). Our results showed a relationship between cholesterol, Pgp activity, and resistance to anthelmintics, although further investigations are needed to understand better the local lipid environment of Pgp and its role in Pgp activity in nematode envelopes.

In conclusion, our results provide, for the first time, detailed information on the lipid composition of nematode eggs and on the relationships between these lipids and xenobiotic transport. The number of Pgp with the active conformation has been correlated with free cholesterol and the PL content of eggs. The resistance to anthelmintics of the nematodes has been positively correlated with the free cholesterol content of eggs. Similar relationships remain to be examined in the other stages of parasite development and in other nematode species. If confirmed, these observations may be exploited for a better understanding of the development of drug resistance in nematodes and for an improved anti-parasitic therapy.

Acknowledgment This work was financed by INRA and the Region Centre. We thank Dr. Yan Van Wyk, Dr. Gilles Aumont, and Professor Roger Prichard for kindly providing the H. contortus isolates, Mr. Limouzin and his team for the careful maintenance of the animals, and Marie-Colette Fauré and her team for their precious help with the references. The experiments comply with the current French laws.

\section{References}

Beaumont-Schwartz C,Kerboeuf D, Hubert J (1987) Méthodes de mise en évidence de souche de strongles gastro-intestinaux resistantes aux anthelminthiques. Rec Med Vet 163:683688

Beech RN, Prichard RK, Scott ME (1994) Genetic variability of the beta-tubulin genes in benzimidazole-susceptible and -resistant strains of Haemonchus contortus. Genetics 138:103-110

Berleur F, Roman V, Jaskierowicz D, Daveloose D, Leterrier F, TerMinassian-Saraga L, Madelmont G (1985) Interaction of vinblastine sulfate with artificial phospholipid membranes: a study by differential scanning calorimetry and spin labeling. Biochem Pharmacol 34:3081-3086

Chao WT, Tsai SH, Lin YC, Lin WW, Yang VC (2005) Cellular localization and interaction of ABCA1 and caveolin-1 in aortic endothelial cells after HDL incubation. Biochem Biophys Res Commun 332:743-749

Chitwood DJ, Lusby WR (1991) Metabolism of plant sterols by nematodes. Lipids 26:619-627

Clarke AJ, Cox PM, Shepherd AM (1967) The chemical composition of the egg shells of the potato cyst-nematode, Heterodera rostochiensis. Woll Biochem J 104:1056-1060

Coles GC (1990) Recent advances in laboratory models for evaluation of helminth chemotherapy. Br Vet J 146:113-119

Crane JM, Tamm LK (2004) Role of cholesterol in the formation and nature of lipid rafts in planar and spherical model membranes. Biophys J 86:2965-2979

Douard V, Hermier D, Blesbois E (2000) Changes in turkey semen lipids during liquid in vitro storage. Biol Reprod 63:1450-1456

Flesch FM, Brouwers JF, Nievelstein PF, Verkleij AJ, van Golde LM, Colenbrander B, Gadella BM (2001) Bicarbonate stimulated phospholipid scrambling induces cholesterol redistribution and enables cholesterol depletion in the sperm plasma membrane. J Cell Sci 114:3543-3555

Garrigues A, Escargueil AE, Orlowski S (2002) The multidrug transporter, $P$-glycoprotein, actively mediates cholesterol redistribution in the cell membrane. Proc Natl Acad Sci USA 99:10347-10352

Ghetie MA, Marches R, Kufert S, Vitetta ES (2004) An anti-CD19 antibody inhibits the interaction between $P$-glycoprotein $(P$-gp $)$ and CD19, causes $P$-gp to translocate out of lipid rafts, and chemosensitizes a multidrug-resistant (MDR) lymphoma cell line. Blood 104:178-183

Gibbons LM (1979) Revision of the genus Haemonchus Cobb, 1898 (Nematoda, Trichostrongylidae). Syst Parasitol 1:3-24

Goormaghtigh E, Chatelain P, Caspers J, Ruysschaert JM (1980) Evidence of a complex between adriamycin derivatives and cardiolipin: possible role in cardiotoxicity. Biochem Pharmacol 29:3003-3010

Hieb WF, Rothstein, M (1968) Sterol requirement for reproduction of a free-living nematode. Science 160:778-780

Kapur J, Sood ML (1985) Haemonchus contortus: qualitative and quantitative analysis of lipids. Zentralbl Veterinarmed B 32:345353

Kapur J, Sood ML (1987) Biochemistry of Haemonchus - a review. Angew Parasitol 28:211-228

Kerboeuf D, Chambrier P, Le Vern Y, Aycardi J (1999) Flow cytometry analysis of drug transport mechanisms in Haemonchus contortus susceptible or resistant to anthelmintics. Parasitol Res $85: 118-123$

Kerboeuf D, Guegnard F, Le Vern Y (2002) Analysis and partial reversal of multidrug resistance to anthelmintics due to $P$ glycoprotein in Haemonchus contortus eggs using Lens culinaris lectin. Parasitol Res 88:816-821 
Kerboeuf D, Blackhall W, Kaminsky R, von Samson-Himmelstjerna G (2003a) $P$-glycoprotein in helminths: function and perspectives for anthelmintic treatment and reversal of resistance. Int $\mathrm{J}$ Antimicrob Agents 22:332-346

Kerboeuf D, Guegnard F, Vern YL (2003b) Detection of $P$ glycoprotein-mediated multidrug resistance against anthelmintics in Haemonchus contortus using anti-human mdr1 monoclonal antibodies. Parasitol Res 91:79-85

Klein U, Gimpl G, Fahrenholz F (1995) Alteration of the myometrial plasma membrane cholesterol content with beta-cyclodextrin modulates the binding affinity of the oxytocin receptor. Biochemistry (Mosc) 34:13784-13793

Klein C, Pillot T, Chambaz J, Drouet B (2003) Determination of plasma membrane fluidity with a fluorescent analogue of sphingomyelin by FRAP measurement using a standard confocal microscope. Brain Res Protoc 11:46-51

Kremer JJ, Sklansky DJ, Murphy RM (2001) Profile of changes in lipid bilayer structure caused by beta-amyloid peptide. Biochemistry (Mosc) 40:8563-8571

Kurzchalia TV, Ward S (2003) Why do worms need cholesterol? Nat Cell Biol 5:684-688

Kwa MS, Kooyman FN, Boersema JH, Roos MH (1993) Effect of selection for benzimidazole resistance in Haemonchus contortus on beta-tubulin isotype 1 and isotype 2 genes. Biochem Biophys Res Commun 191:413-419

Laird DM, Eble KS, Cunningham CC (1986a) Reconstitution of mitochondrial F0F1-ATPase with phosphatidylcholine using the nonionic detergent, octylglucoside. J Biol Chem 261:14844-14850

Laird DM, Parce JW, Montgomery RI, Cunningham CC (1986b) Effect of phospholipids on the catalytic subunits of the mitochondrial F0F1-ATPase. J Biol Chem 261:14851-14856

Maloney MD, Semprevivo LH (1991) Thin-layer and liquid column chromatographic analyses of the lipids of adult Onchocerca gibsoni. Parasitol Res 77:294-300

Manifava M, Thuring JW, Lim ZY, Packman L, Holmes AB, Ktistakis NT (2001) Differential binding of traffic-related proteins to phosphatidic acid- or phosphatidylinositol $(4,5)$ - bisphosphatecoupled affinity reagents. J Biol Chem 276:8987-8994

Mansfield LS, Gamble HR, Fetterer RH (1992) Characterization of the eggshell of Haemonchus contortus-I, Structural components. Comp Biochem Physiol B Biochem Mol Biol 103:681-686

Matyash V, Geier C, Henske A, Mukherjee S, Hirsh D, Thiele C, Grant B, Maxfield FR, Kurzchalia TV (2001) Distribution and transport of cholesterol in Caenorhabditis elegans. Mol Biol Cell 12:1725-1736

McConnell HM, Vrljic M (2003) Liquid-liquid immiscibility in membranes. Annu Rev Biophys Biomol Struct 32:469-492

McGlynn R, Dobrenis K, Walkley SU (2004) Differential subcellular localization of cholesterol, gangliosides, and glycosaminoglycans in murine models of mucopolysaccharide storage disorders. J Comp Neurol 480:415-426

Mechetner EB, Roninson IB (1992) Efficient inhibition of $P$ glycoprotein-mediated multidrug resistance with a monoclonal antibody. Proc Natl Acad Sci USA 89:5824-5828

Mehlhorn H (2001) Encyclopedic reference of parasitology, biologystructure-function. Springer, Berlin Heidelberg New York, p 672

Ohvo H, Slotte JP (1996) Cyclodextrin-mediated removal of sterols from monolayers: effects of sterol structure and phospholipids on desorption rate. Biochemistry (Mosc) 35:8018-8024

Oram JF, Wolfbauer G, Vaughan AM, Tang C, Albers JJ (2003) Phospholipid transfer protein interacts with and stabilizes ATPbinding cassette transporter $\mathrm{A} 1$ and enhances cholesterol efflux from cells. J Biol Chem 278:52379-52385
Osterberg T, Svensson M, Lundahl P (2001) Chromatographic retention of drug molecules on immobilised liposomes prepared from egg phospholipids and from chemically pure phospholipids. Eur J Pharm Sci 12:427-439

Pallares-Trujillo J, Lopez-Soriano FJ, Argiles JM (2000) Lipids: a key role in multidrug resistance? (Review). Int J Oncol 16:783-798

Pohl A, Lage H, Muller P, Pomorski T, Herrmann A (2002) Transport of phosphatidylserine via MDR1 (multidrug resistance 1) $P$ glycoprotein in a human gastric carcinoma cell line. Biochem $\mathrm{J}$ 365:259-268

Radhakrishna H, Donaldson JG (1997) ADP-ribosylation factor 6 regulates a novel plasma membrane recycling pathway. J Cell Biol 139:49-61

Raggers RJ, Pomorski T, Holthuis JC, Kalin N, van Meer G (2000) Lipid traffic: the $\mathrm{ABC}$ of transbilayer movement. Traffic 1:226234

Riou M, Guegnard F, Le Vern Y, Kerboeuf D (2003) Modulation of the multidrug resistance (MDR) system in the nematode Haemonchus contortus by changing cholesterol content: effects on resistance to anthelmintic. J Antimicrob Chemother 52:180 187

Riou M, Koch C, Delaleu B, Berthon P, Kerboeuf D (2005a) Immunolocalisation of an $\mathrm{ABC}$ transporter, $P$-glycoprotein, in the eggshells and cuticles of free-living and parasitic stages of Haemonchus contortus. Parasitol Res 96:142-148

Riou M, Koch C, Kerboeuf D (2005b) Increased resistance to anthelmintics of Haemonchus contortus eggs associated with changes in membrane fluidity of eggshells during embryonation. Parasitol Res 95:266-272

Rothnie A, Theron D, Soceneantu L, Martin C, Traikia M, Berridge G, Higgins CF, Devaux PF, Callaghan R (2001) The importance of cholesterol in maintenance of $P$-glycoprotein activity and its membrane perturbing influence. Eur Biophys J 30:430-442

Sarwal R, Sanyal SN, Khera S (1989) Lipid metabolism in Trichuris globulosa (Nematoda). J Helminthol 63:287-297

Shechter E, Rossignol B (1997) Biochimie et biophysique des membranes Aspects structuraux et fonctionnels. Dunod, Paris, France, $\mathrm{p} 459$

Shio H, Haley NJ, Fowler S (1979) Characterization of lipid-laden aortic cells from cholesterol-fed rabbits, III. Intracellular localization of cholesterol and cholesteryl ester. Lab Invest 41:160 167

Sinicrope FA, Dudeja PK, Bissonnette BM, Safa AR, Brasitus TA (1992) Modulation of $P$-glycoprotein-mediated drug transport by alterations in lipid fluidity of rat liver canalicular membrane vesicles. J Biol Chem 267:24995-25002

Speelmans G, Sips WH, Grisel RJ, Staffhorst RW, Fichtinger-Schepman AM, Reedijk J, de Kruijff B (1996) The interaction of the anticancer drug cisplatin with phospholipids is specific for negatively charged phospholipids and takes place at low chloride ion concentration. Biochim Biophys Acta 1283:60-66

Surai PF, Brillard JP, Speake BK, Blesbois E, Seigneurin F, Sparks NH (2000) Phospholipid fatty acid composition, vitamin E content and susceptibility to lipid peroxidation of duck spermatozoa. Theriogenology 53:1025-1039

Visconti PE, Ning X, Fornes MW, Alvarez JG, Stein P, Connors SA, Kopf GS (1999) Cholesterol efflux-mediated signal transduction in mammalian sperm: cholesterol release signals an increase in protein tyrosine phosphorylation during mouse sperm capacitation. Dev Biol 214:429-443

Zeng Y, Han X, Gross RW (1999) Phospholipid subclass-specific partitioning of lipophilic ions in membrane-water systems. Biochem J 338(Pt 3):651-658 